\title{
Validation and clinical interpretation of the St George's respiratory questionnaire for COPD (SGRQ-C) after adaptation to Malaysian language and culture, in patients with COPD
}

\author{
Anees ur Rehman ${ }^{1,2^{*}}$ (D), Mohamed Azmi Ahmad Hassali ${ }^{1}$, Sabariah Noor Harun ${ }^{1}$, Sameen Abbas $^{3}$,
} Jaya Muneswarao ${ }^{1}$, Irfhan Ali Bin Hyder Ali $^{4}$ and Rabia Hussain ${ }^{1}$

\begin{abstract}
Background: Cultural differences affect the administration and results of health status questionnaires. "Cross cultural adaptation" ensures retention of psychometric properties such as validity and reliability at an item and/or scale level.

Objective: To develop a Malaysian version of St George's respiratory COPD specific questionnaire (SGRQ-CM), to evaluate the full spectrum of psychometric properties (reliability, validity and responsiveness), to test the factor structure and to assess minimum clinically important difference for the SGRQ-CM, to be used in population of Malaysia.

Methodology: SGRQ-C was translated to Bahasa Malaysia using a standard protocol. 240 COPD patients were included in the study. All patients were followed-up for six months. Construct validity, internal consistency, item convergent validity, test-retest ability, responsiveness, factor analysis and MCID of the Malaysian version of SGRQ-C to be used in population of Malaysia were evaluated.

Results: The Cronbach alpha coefficient and intraclass correlation coefficients (ICC) for SGRQ-CM were reported as 0.87 , and 0.88 respectively. Correlation of SGRQ-CM with CAT, EQ-5D- 5 L, mMRC dyspnea scales and FEV ${ }_{1} \%$ predicted were reported as $0.86,-0.82,0.72$ and -0.42 respectively. Correlation coefficient between the subscales and other clinical and health status measures ranged from $r=-0.35$ to $r=-0.87$. The MCID was reported as $5.07(-2.54-12.67)$.

Conclusion: The Malaysian version of SGRQ-C has a good psychometric property comparable to those of the original version and has a strong evidence of validity, reliability and responsiveness towards disease severity in Malaysian COPD patients. It can be recommended as a reliable quality of life measure for future research.
\end{abstract}

Keywords: SGRQ-C, COPD, MCID, Psychometric validation, Quality of life

\footnotetext{
* Correspondence: aneesurrehmanr90@gmail.com

'Department of Clinical Pharmacy, School of Pharmaceutical Sciences,

University Sains Malaysia, Minden, 11800 Penang, Malaysia

${ }^{2}$ Faculty of Pharmacy, Bahauddin Zakariya University, Multan, Pakistan

Full list of author information is available at the end of the article
}

(c) The Author(s). 2020 Open Access This article is licensed under a Creative Commons Attribution 4.0 International License, which permits use, sharing, adaptation, distribution and reproduction in any medium or format, as long as you give appropriate credit to the original author(s) and the source, provide a link to the Creative Commons licence, and indicate if changes were made. The images or other third party material in this article are included in the article's Creative Commons licence, unless indicated otherwise in a credit line to the material. If material is not included in the article's Creative Commons licence and your intended use is not permitted by statutory regulation or exceeds the permitted use, you will need to obtain permission directly from the copyright holder. To view a copy of this licence, visit http://creativecommons.org/licenses/by/4.0/ The Creative Commons Public Domain Dedication waiver (http://creativecommons.org/publicdomain/zero/1.0/) applies to the data made available in this article, unless otherwise stated in a credit line to the data. 


\section{Key Points}

1. SGRQ-CM has a strong evidence of validity (construct and concurrent), reliability and responsiveness to disease severity in Malaysian COPD patients.

2. It can be used as a reliable QoL measure in future research, including randomized clinical trials, rehabilitation studies, and QoL studies in COPD patients.

3. It can be used in clinical practice to measure treatment efficacy for selection of optimal treatment.

\section{Introduction}

In addition to spirometric values, Global Initiative for Chronic Obstructive Lung Disease (GOLD) 2019 updates recommend to assess impact of chronic obstructive lung disease (COPD) on the health status of patients, disease progression rate and exacerbation frequency prior to initiation of new therapy [1]. COPD is a multifactorial health problem, having substantial impact on patients' financial status and quality of life (QoL) $[2,3]$. COPD patients experience numerous pulmonary and extra pulmonary symptoms, including dyspnea, cough, sputum, fatigue, insomnia and systemic inflammation. These symptoms are responsible for impaired QoL and can be assessed through communication with the patient rather than spirometry. Disease activity markers (lung function's parameters, sputum frequency, sputum volume and exacerbation), may not necessarily reflect the overall impact of the disease on an individual patient [4]. Incorporating health status questionnaires into the clinical investigations of COPD may aid in determining the treatment efficacy, disease severity and health status of COPD patients [4].

In general, QoL questionnaires are widely used to observe patient improvement, responsiveness to ongoing therapy, verify the lab test values, and effect of clinical interventions. They are also helpful in the selection of most appropriate therapy among a broad spectrum of therapeutic interventions. The SGRQ-C is a selfadministered COPD specific questionnaire to evaluate the comparative measurement of health status based on severity of disease and can also evaluate the effectiveness of therapy after treatment [5]. It has adequate sensitivity and reliability. Once efficiently translated and validated, it provides a standard metric across cultures and populations [6-9]. It can evaluate disease symptoms, patient's daily activity, the impact of COPD on patient's life and total QoL score. Each item in the SGRQ-C questionnaire has a specific weight, which is combined to calculate subscale or total score [5]. The minimum clinically important difference (MCID) for the SGRQ-C is its response to therapeutic interventions and is used to evaluate clinical efficacy of the therapy in COPD patients. MCID is valid and correlates with clinical parameters of respiratory function e.g. forced expiratory volume in one second $\left(\mathrm{FEV}_{1}\right)$ [10].

Cultural differences affect the administration and results of health status questionnaires [11]. Food and Drug Administration Authority (FDA) requires validation of patient reported health status measures in terms of linguistic and cultural adaptation to ensure content validity at the conceptual level among different populations before applying to a new or different population [12]. "Cross cultural adaptation" resolves translation and cultural adaptation issues in using a questionnaire in different ethnicity than the source of origin, and finds content similarity between source and target population [13]. It ensures retention of psychometric properties such as validity and reliability at an item and/or a scale level. Moreover, the translation of the questionnaire to native language not only improves communication between patient and healthcare professionals, but also helps in measuring the exact experience of individuals on QoL due to influence of the disease without any interference from the health professional [14].

Currently modified medical research council dyspnea scale (mMRC scale), COPD assessment test (CAT) and few generic health status questionnaires e.g. European Quality of life 5-Dimension 5-Level questionnaire (EQ5D-5 L) are being used in clinical practice in Malaysia. CAT is a short form of SGRQ-C and can only measure respiratory disability and few activities among patients with COPD [15]. Whereas, generic questionnaires are less responsive to changes in disease status and are less effective for use in clinical trials as they do not focus on aspect, specific to a certain disease $[16,17]$. There is a need for a scale which can measure in detail the four main domains of health status that include physiological functioning, symptoms, functional impairment, and QoL. Translation and validation of SGRQ-C to Malaysian language and culture can cover this gap, as it can assess the detailed symptomatic effect of disease and impaired QoL among COPD patients in clinical practice and research studies. Bahasa Malaysia is an official language of Malaysia and is spoken by approximately $85 \%$ of the Malaysian population [18]. The objective of our study was to develop a Malaysian version of SGRQ-C (SGRQ-CM), to evaluate the full spectrum of psychometric properties (reliability, validity and responsiveness) of the SGRQ-CM, to test the factor structure of SGRQCM and to assess MCID for the SGRQ-CM, to be used in population of Malaysia.

\section{Methodology}

This study was part of a prospective cohort that included patients from the main public hospital of Penang, 
Malaysia. The study protocol was approved by the National Medical Research Register Malaysia and clinical research center of concerned hospital (Registration number: NMRR-18-1482-42,075). Sample size for validation of the SGRQ-C questionnaire was calculated as 240 (5 participants against each item in the instrument with $20 \%$ drop out rate) [19]. Written informed consent was obtained from all participants.

\section{Translation}

The translated version of the SGRQ-C questionnaire was available from the St George's library. To verify the translated version, a native Malay speaker with good command on English was selected and performed forward translation of SGRQ-C English version to Malaysian language.

A team comprising medical experts, academicians and the translator reviewed the both translated versions of SGRQ-C and developed a new translated version by resolving conflicts through discussion. To check the validity of the newly Malaysian translated version that it reflects the same content as original version, two independent translators translated the newly Malaysian translated version to the English. Then a committee of experts comprising health professionals, academicians and translators (forward translator and backward translators) reviewed the forward and backward translated version to come up with a final version of Malaysian translated SGRQ-C.

The translated version was reviewed critically to validate semantic, idiomatic, experiential and conceptual equivalence. To assess that the translated version still retains its equivalence to the original version, it was filled initially by 10 COPD patients and the patients were interviewed to probe what he/she thought about the meaning of each questionnaire. No difficulty was reported in understanding or answering the questions. Therefore, the Malaysian version of SGRQ-C (SGRQ$\mathrm{CM}$ ) was adopted as the final version of the questionnaire (Fig. 1).

\section{Study design}

Data on clinical and health status of COPD patients was collected from the patients visiting chest clinic of the Penang Hospital. Patients were followed up for six months. Data were collected for all the included patients

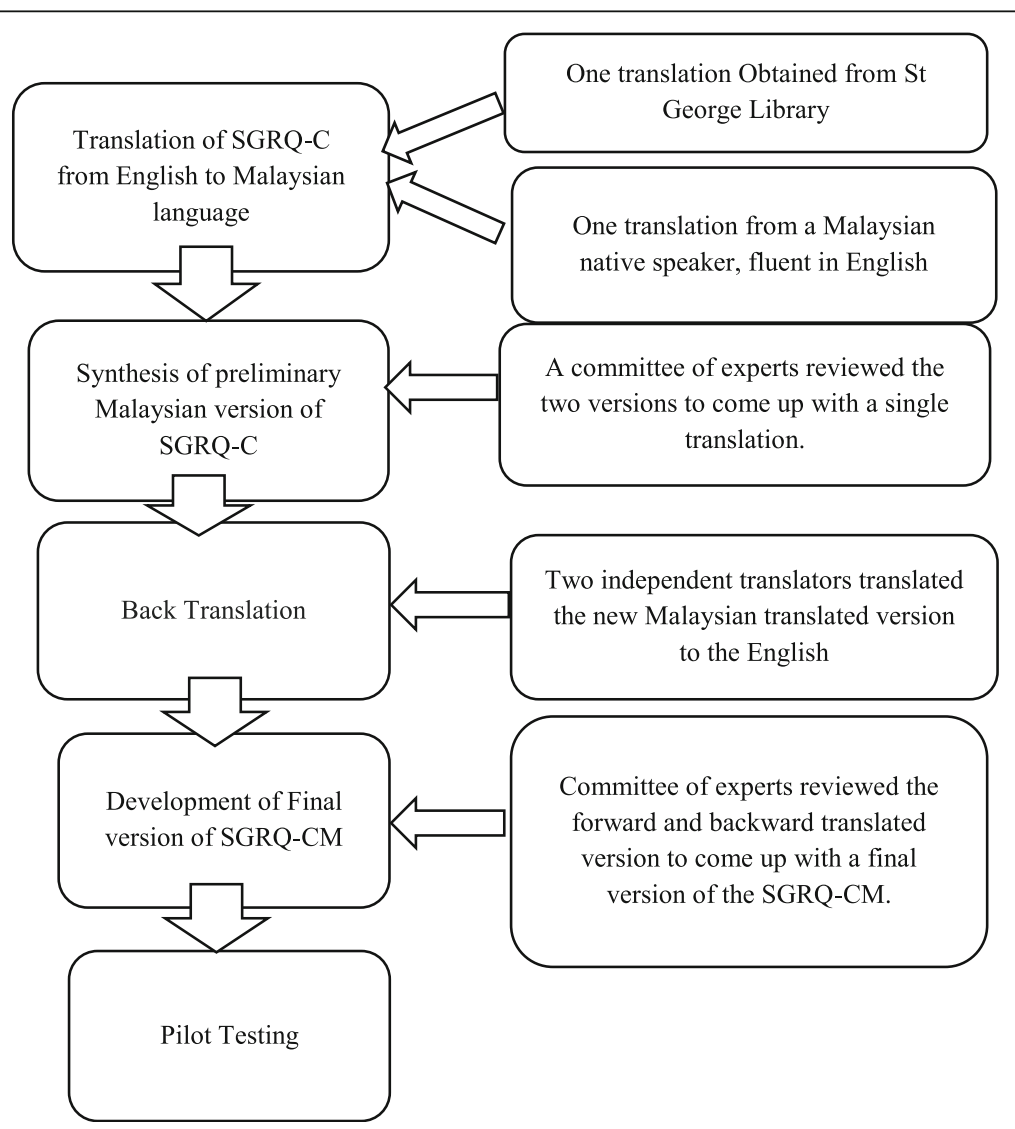

Fig. 1 Translation and linguistic validation methodology used for translation of the SGRQ-C. ${ }^{*} S G R Q-C$, St George Respiratory Questionnaire for COPD; SGRQ-CM, Malaysian version of SGRQ-C 
at baseline during recruitment, then a repeated evaluation of $20 \%$ patients at an interval of two weeks and then follow-up evaluation of all patients at an interval of six months.

Among 240 patients included in the study forty seven patients suffered from exacerbation and admitted to the ward during the study period. For admitted patients, data were also collected on the last day of admission due to exacerbation and at an interval of one month after discharge when a significant improvement in health status was expected.

The patients were included in the study if they had, (1) confirmed diagnosis of COPD $\left(\mathrm{FEV}_{1} / \mathrm{FVC}<70\right)$, (2) minimum of six-month outpatient record to avoid abrupt changes in QoL due to initiation of therapy, (3) no changes in treatment over the past 4 weeks, (4) no other respiratory disorders, (5) no other diseases that have a short term effect on QoL, and (6) no disability. Patients were clinically assessed and then administered the data collection form consisting of demographic data, clinical data, and health status questionnaires (SGRQCM, CAT, EQ-5D-5 L, and mMRC dyspnea scale). Help was provided by non-technical staff to complete the questionnaire, if someone was unable to complete by himself due to poor eyesight, unable to read or shaky hands. Nondirective guidance was provided if patients had queries on how to answer questions.

For SGRQ-CM questionnaire the recall period for symptom and activity subscale was "past year" and "these days" respectively. Spirometry was performed according to American Thoracic society guidelines at each visit before administration of the data collection form. Severity of COPD was categorized according to spirometry results, in accordance with GOLD 2018 guidelines [20]. Grade I COPD with $\mathrm{FEV}_{1} \geq 80 \%$ predicted, grade II with $\mathrm{FEV}_{1} 50$ to $80 \%$ predicted, grade III at $\mathrm{FEV}_{1} 30$ to $50 \%$ predicted, and grade IV with $\mathrm{FEV}_{1}<30 \%$ predicted. Stable COPD patients were defined as patients having less than $10 \%$ change in spirometric values, and no variation in clinical symptoms after two weeks.

\section{Health status measures}

Three health status measures, CAT, the Malaysian version of EQ-5D-5 L, and mMRC dyspnea scale were simultaneously applied to the study population along with the SGRQ-CM. CAT is an FDA approved health status questionnaire used for assessment of COPD patients. CAT is easy to understand and consist of 8 items related to symptoms and activities. Each item has scores 0 to 5 from best to worst with a maximum total score of 40. Its reliability has been already tested in many European countries and literature shows the high correlation of SGRQ-C score and CAT score [21].
The EQ-5D-5 L is a generic health status measure comprising two components: a descriptive utility index (EQ-5D-5 L UI) and a visual analogue scale (EQ-VAS) [22]. The utility index is calculated from the descriptive scale of five components (mobility, self-care, usual activities, pain and depression). Patients mark each dimension on a scale of 1 "no problem" to 5 "worst problem". The five-digit number (ranging from 11,111 to 55,555) obtained was then converted to a utility index based on EQ-5D-5 L value set for Malaysia [23]. For EQ-VAS participants were asked to mark their health on a vertical visual analogue scale from 0 (worst possible health) to 100 (best possible health). Higher EQ-5D-5 L UI and EQ-VAS values reflect good health status.

The mMRC is a self-administered FDA approved health status measure. It has been used for years to assess the level of breathlessness and its impact on daily activities on a scale from 0 to 4 (Grade 0: not troubled by breathlessness except on strenuous exercise, Grade 1: Short of breath when hurrying or walking up a slight hill, Grade 2: walks slower than contemporaries on level ground because of breathlessness, Grade 3: Stops for breath after walking about $100 \mathrm{~m}$ (109 yards) or after a few minutes on level ground, Grade 4: too breathless to leave the house or breathless when dressing or undressing). It is easy to use, and has a well-established relationship with spirometric values and SGRQ-C [24].

\section{Psychometric analyses}

Construct validity (known-group validity and reliability) was assessed by analyzing the correlation of SGRQ-CM total and subscale scores with clinical measures and other health status measures (CAT, EQ-5D-5 L and $\mathrm{mMRC}$ ) obtained during the baseline assessment. The linear trend between SGRQ-CM scores and spirometry values across different levels of disease severity was tested using Pearson's correlation coefficient.

Internal consistency reliability of SGRQ-CM total score, symptom subscale, activity subscale and impact subscale score was assessed based on the value of Cronbach's alpha coefficient. Internal consistency reliability for each scale is considered as excellent if Cronbach's $\alpha$ is $\geq 0.9$, strong if Cronbach's $\alpha$ is $\geq 0.8$, acceptable if Cronbach's $\alpha$ is $\geq 0.7$ and reasonable if Cronbach's $\alpha$ is $\geq 0.6$. This cut of values have been adapted from Taber, K. S. (2018) [25]. Test-retest ability was assessed using intraclass correlation coefficients (ICC) [26]. Responsiveness can be defined as the ability of the health status measure to determine clinically meaningful changes with the passage of time or under the impact of an intervention. The responsiveness of SGRQ-CM was assessed by calculating the effect size (ES). ES determines the magnitude of change and was calculated by using the mean difference and standard deviation (SD) at baseline and 
after six months of follow-up and between patients at different stages of disease severity. The mean difference between two groups was divided by change in SD at two time intervals. The value of $\mathrm{ES}$ of $\geq 0.8, \geq 0.5$ and $\geq 0.2$ represent strong, medium and small sensitivity respectively, and this cut of value was adapted from Cohen, J. (1988) [27].

Factor analysis evaluates the internal structure of a questionnaire. It gives the significance of each item with the hypothesized scales and the overall model fit. The three factor structure of SGRQ-C was confirmed using explanatory factor analysis (EFA) and confirmatory factor analysis (CFA). For EFA the accuracy of the model fit was confirmed with Kaser-Meie Olkan (KMO) value (good fit if $\mathrm{KMO} \geq 0.8$ and $\leq 1.00$; satisfactory if $\mathrm{KMO}$ $\geq 0.7$ and $<0.8$; acceptable if $\mathrm{KMO} \geq 0.6$ and $<0.7$; and unacceptable if $\mathrm{KMO}<0.6)$ and a significant $p$ value of Bartlet's test of sphericity. These cut of points values have been adapted from Li, C.-H. (2016) [28]. In EFA, allocation of an item to the relevant factor was considered satisfactory if, the Item showed factor loading value of $>$ 0.25 to the particular subscale [29]. CFA was then performed to confirm the results of EFA. To access the accuracy of model (model fit) for CFA following fit indices were used: standardized root mean square residual (SRMR), good fit if $S R M R \leq 0.08$ and acceptable if SRMR $>0.08$ to $<0.1$; the root mean square error of approximation (RSMEA), good fit if RSMEA $\leq 0.06$, acceptable fit if RMSEA is $>0.06$ and $<0.08$; the comparative fit index (CFI), good fit if CFI $\geq 0.95$ and acceptable fit if CFI is $\geq 0.90$ and $\leq 0.95$; the Tucker-Lewis index (TLI), good fit if TLI $\geq 0.90$. All these values were adapted from Li, C.-H. (2016) [28]. Scaling success rate was assessed for each subscale as the percentage of items within a subscale that is highly correlated with the hypothesized subscale as compared to other subscales.

MCID indicates a clinical threshold which is used to differentiate between patients and within patients under the impact of an intervention with the passage of time and provides information about an intervention that it achieved the minimum level of perceived benefit and moves beyond the concept of statistical differences [30]. There is no single established method for measurement of MCID. Different approaches are used to assess the MCID. Anchor-based method measures MCID based on relationship between the instrument to be assessed and other measures of health status (the anchors) [31]. Using Anchor-based approach MCID was assessed by calculating the mean difference $(95 \% \mathrm{CI})$ in baseline SGRQ-CM total and impact subscale scores among patients at adjacent disease and dyspnea level. The difference between dyspnea grade III and IV is important, as it distinguish between patients with no activity from patients with comparably better activity. Distribution-based approach involves the comparison of health status score to a clear change in patient clinical status or to a criterion of health which requires major change in treatment. The principle underlying this approach is that health status measure scores should be worst in patients underlying major health event and will improve significantly with the recovery form the event [31]. Exacerbation causes significant changes in patient's clinical status and spirometric values and $75 \%$ recovery is expected after exacerbation in the first four weeks, which indicates a major improvement in health status [9]. Using distributionbased approach MCID was assessed by administering the questionnaire to the COPD patients being discharged from hospital after exacerbation and at onemonth post-exacerbation treatment when a significant improvement in health status was expected.

It was hypothesized that SGRQ-CM would differentiate patients on the basis of their disease severity (known-group validity), has a significant correlations with clinical and health status measures (CAT, mMRC dysponea scale, EQ-5D-5 L and spirometric values) (reliability), and can detect changes in QoL under the influence of disease over time (responsiveness) [32, 33]. A $p$ value less than 0.05 (two sided) was considered to be statistically significant. All analysis were performed using SPSS version 24.0 (IBM SPSS Statistics for Windows, 142 Armonk, NY: IBM Corp.) and IBM AMOS version 20.0 (Arbuckle, JL, Chicago: IBM SPSS).

\section{Results}

A total of 240 patients who met the inclusion criteria were included in the study. Mean age was reported as 69.40 years, and mean BMI was reported as 23.49. Among the included patients $44.2 \%$ were Chinese, $24.5 \%$ were Malay, and $29.2 \%$ were Indian. $89.5 \%$ of the patients were current or ex-smoker. At baseline mean FEV1/FVC and $\mathrm{FEV}_{1} \%$ predicted were reported as 51.72 and 55.27 respectively. The mean SGRQ-CM score was reported as 49.93. The mean SGRQ-CM scores in GOLD 1, GOLD 2, GOLD 3, and GOLD 4 patients were reported as $41.89,45.26,53.25$ and 64.30 respectively. Demographic characteristics and clinical measures of the sample population are shown in the Table 1 .

EFA results showed that 3-factor structure of SGRQCM was optimal, explaining $68.31 \%$ of the variance. In EFA, the adequacy of the factor model was confirmed, with KMO value of 0.759 and a significant $p$ value of Bartlet's test of sphericity $(p<0.001)$. Loading of SGRQ$\mathrm{CM}$ item 2, 3, 6, 9.5, 12.3, 10.6, 14, and 11.5 were higher than 0.25 in more than one factors, the factor with highest loading was selected [34]. In EFA Six out of seven symptom items showed loading in symptom factor with a success rate of $87.5 \%$. Similarly loading of relative items in Activity and impact factor showed a success 
Table 1 Demographic and clinical characteristics of COPD patients at baseline

\begin{tabular}{|c|c|}
\hline & Outpatient (Stable Disease) $n=240$ \\
\hline Age: mean Years (SD) & $69.40(6.87) \mathrm{kg} / \mathrm{m}^{2}$ \\
\hline BMI: mean (SD) & $23.49(4.35)$ \\
\hline \multicolumn{2}{|l|}{ Ethnicity: \% (n) } \\
\hline Malay & $24.5(60)$ \\
\hline Chinese & $44.2(106)$ \\
\hline Tamil & $29.2(69)$ \\
\hline Others & $2.08(5)$ \\
\hline \multicolumn{2}{|l|}{ Smoking Status: \% (n) } \\
\hline Current Smoker & $11.6(28)$ \\
\hline Ex-Smoker & $77.9(187)$ \\
\hline Non-Smoker & $10.4(25)$ \\
\hline \multicolumn{2}{|c|}{ Post-bronchodilator Spirometry: Mean (SD) } \\
\hline $\mathrm{FEV}_{1} / \mathrm{FVC}$ & $51.72(12.75)$ \\
\hline FEV1 (\%) & $55.27(14.84)$ \\
\hline \multicolumn{2}{|c|}{$\begin{array}{l}\text { Mean }(95 \% \mathrm{CI}) \text { SGRQ-CM scores at different disease stage: GOLD } \\
\text { Classification (\% Predicted FEV } 1 \text { ) }\end{array}$} \\
\hline I (> 80\%) & $41.89(29.45-54.34)$ \\
\hline || (50-79\%) & $45.26(41.32-49.19)$ \\
\hline |II (30-49\%) & $53.52(47.52-59.52)$ \\
\hline IV $(<30 \%)$ & $64.30(56.72-71.87)$ \\
\hline
\end{tabular}

Mean (95\% CI)SGRQ-CM scores at different disease stage: mMRC Dyspnea grade

$\begin{array}{ll}\text { Grade } 0 & 29.24(25.9-32.58) \\ \text { Grade } 1 & 32.46(29-35.91) \\ \text { Grade 2 } & 47.90(42.2-53.57) \\ \text { Grade 3 } & 57.77(53.3-62.16) \\ \text { Grade 4 } & 66.36(60.8-71.85)\end{array}$

Health status instrument Score: mean (SD)

$\begin{array}{ll}\text { CAT } & 16.52(6.85) \\ \text { EQ-5D-5 L UI } & 0.62(0.23) \\ \text { EQ-VAS } & 59.24(17.30) \\ \text { mMRC } & 2.31(1.31)\end{array}$

SGRQ-CM Scores: mean (SD)

$\begin{array}{ll}\text { Symptom } & 55.44(19.98) \\ \text { Activity } & 58.43(18.78) \\ \text { Impact } & 43(20.71) \\ \text { Total Score } & 49.93(18.61)\end{array}$

$B M I$ Body mass index in $\mathrm{kg} / \mathrm{m}^{2}, C O P D$ Chronic Obstructive Pulmonary Disease, CAT COPD assessment test, EQ-5D-5 L UI European Quality of life 5-Dimension 5-Level questionnaire Utility index score, EQ-VAS European Quality of life 5Dimension 5-Level questionnaire Visual analogue score, $F E V{ }_{1}$ Forced expiratory volume in $1 \mathrm{~s}, F V C$ Forced vital capacity, GOLD Global Initiative for Chronic Obstructive Lung Disease, $m M R C$ modified Medical Research Council dyspnea scale, SGRQ-CM St George's Respiratory COPD specific Questionnaire Malaysian version, SD Standard deviation rate of $84.6 \%$ (11 out of 13 items) and $80 \%$ (16 out of 20 items) respectively. CFA demonstrated reasonable model fit with, SRMR $=0.058$, RMSEA $=0.052, \mathrm{CFI}=0.921$ and $\mathrm{TLI}=0.910$. In CFA factor loading value ranged from 0.408 to 0.765 . In CFA all the item loaded to their respective scales with a success rate of $100 \%$. Table 2 shows the loading of SGRQ-CM in 3 factor structure model.

Scaling properties, internal consistency and test-retest reliability results are presented in Table 3. Approximately $2.1 \%$ of patients showed the best possible score in symptom components and $4.3 \%$ of patients showed the best possible score in activity component. None of the patients showed worst possible score. SGRQ-CM and three subscales showed excellent internal consistency. The Cronbach alpha coefficient for SGRQCM total score, symptom subscale, activity subscale and impact subscale was $0.87,0.74,0.70$ and 0.71 respectively. No significant difference was observed for SGRQ$\mathrm{CM}$ total and subscale scores at baseline and at 2 weeks visit $(p>0.05)$. At two weeks interval, ICC for total SGRQ-CM, symptom subscale, activity subscale and impact subscale was reported as $0.88,0.89,0.85$ and 0.90 respectively.

As shown in Table 4, SGRQ-CM showed good correlation with the CAT, EQ-5D-5L UI, EQ-VAS and mMRC dyspnea scale and low but significant correlation with $\mathrm{FEV}_{1}$ \%predicted in the expected direction. Correlation of SGRQ-CM with CAT, EQ-5D-5 L UI, EQ-VAS, mMRC dyspnea scale and $\mathrm{FEV}_{1} \%$ predicted was reported as $0.86,-0.82,-0.61,0.72$ and -0.42 respectively. Correlation coefficient among the subscales and other clinical and health status measures ranged from $r=-0.31$ to $\mathrm{r}=-0.87$.

Responsiveness of the SGRQ-CM to detect changes in health status over time was presented in Table 5. Patients were followed up for six months. Among 240 included patients twenty seven patients lost the follow-up due to different reasons (changed hospital, moved from the city and surgery). 213 patients completed the follow up visits. The mean difference in $\%$ predicted $\mathrm{FEV}_{1}$ values between baseline and 6 months follow-up was insignificant $(p=0.13)$, whereas, a significant difference was observed in the mean differences of SGRQ-CM total and subscale scores between the two occasions $(p<$ $0.001)$.

Significant mean difference $(95 \% \mathrm{CI})$ was observed in total and component scores of the patients at different levels of disease severity [GOLD IV vs III, 10.78 (1.3820.17), $p=0.02$; and GOLD III vs II, 8.26 (1.27-15.26), $p<0.01]$. The mean difference $(95 \% \mathrm{CI})$ in SGRQ-CM scores in patients at mMRC dyspnea grade IV vs III was 8.59 (2.59-14.59), $\mathrm{p}<0.01$ (Table 6). MCID using anchor-based approach was reported as the mean 
Table 2 Exploratory factor Loadings for the Malaysian version of St George Respiratory COPD specific questionnaire (SGRQ-CM)

Variables
Q1: I cough
Q2: Phlegm was expelled when I cough
Q3: I have shortness of breath
Q4: I have attacks of wheezing
Q5: How many attacks of chest problem did you have during the last year?
Q6: How often do you have good days (with not many chest problems)?
Q7: If you experience wheezing, is it worse in the morning?

\section{Explanatory Factor Analysis}

(EFA)

Activity

Q9.1: Make you feel breathless getting washed or dressed

Q9.2: Make you feel breathless walking around the home

Q9.3: Make you feel breathless walking outside on flat ground

Q9.4: Make you feel breathless walking up a flight of stairs

Q9.5: Make you feel breathless walking up hills

Q12.1: I take a long time to take a bath or put on clothes

Q12.2: I cannot take a bath or shower, or I take a long time

Q12.3: I walk slower than other people, or I stop for rests

Q12.4: Jobs such as housework take a long time, or I have to stop for rests

Q12.5: If I walk up one flight of stairs, I have to go slowly or stop

Q12.6: If I hurry or walk fast, I have to stop or slow down

Q12.7: My breathing makes it difficult to do things such as walk up hills, carrying things upstairs, light gardening such as weeding, dance, play bowling or play golf

Q12.8: My breathing makes it difficult to do things such as carry heavy stuff, dig the garden or gardening, jog or walk at 5 miles per hour, play tennis or swim

Q8: How would you describe your chest condition?

Q10.1: My cough hurts

Q10.2: My cough makes me tired

Q10.3: I am breathless when I talk

Q10.4: I am breathless when I bend over

Q10.5: My cough or breathing disturbs my sleep

Q10.6: I get exhausted easily

Q11.1: My cough or breathing is embarrassing in public

Q11.2: My chest problem is a nuisance to my family, friends or neighbors

Q11.3: I get scared or panic when I cannot breathe

Q11.4: I feel that I am not in control of my chest problem

Q11.5: I have become weak or an invalid due to my chest problems

Q11.6: Exercise is not safe for me

Q11.7: Everything seems too much of an effort

Q13.1: I cannot play sports or games

Q13.2: I cannot go out for entertainment or recreation

Q13.3: I cannot go out of the house to do the shopping

Q13.4: I cannot do housework

0.414
0.376

0.376

Symptoms Impact

(CFA)

0.577

0.473

0.450

0.640

0.517

$$
0.700
$$

0.473

0.329

0.568

0.568

0.284

0.371

0.488

0.621

0.517

0.352

0.48

0.487

0.477

0.398

0.568

0.587

0.516

0.454

0.384

0.270

0.416

0.451

0.510

0.632

0.375

0.514

0.563

0.538

0.302

0.464

0.289

0.533

0.364

0.565

0.408

0.434

0.731

0.445

0.508

0.615

0.663

0.380

0.512

0.343

0.459

0.486

0.429

0.624

0.497

0.439

Q13.5: I cannot move far from my bed or chair

0.360

$-0.659$

$-0.299$

0.568 
Table 3 Results from tests of scaling properties of Malaysian translated version of the St George's Respiratory COPD specific Questionnaire

\begin{tabular}{|c|c|c|c|c|}
\hline & Symptom & Activity & Impact & SGRQ-CM \\
\hline Items $\mathrm{n}$ & 7 & 13 & 20 & 40 \\
\hline Theoretical Range & $0-100$ & $0-100$ & $0-100$ & $0-100$ \\
\hline Observed range & $8.88-100$ & 22.39-100 & $9.23-90.61$ & $17.59-92.32$ \\
\hline At ceiling $\%(n)^{\text {a }}$ & $2.1(3)$ & $4.3(6)$ & 0 & 0 \\
\hline At Floor $\%{ }^{b}$ & 0 & 0 & 0 & 0 \\
\hline Item Convergent Validity ${ }^{c}$ & $0.44-0.79$ & $0.14-0.72$ & $0.13-0.68$ & - \\
\hline Scaling Success $\%^{d}$ & 87.5 & 84.6 & 80 & - \\
\hline Cronbach's a Coefficient & 0.74 & 0.70 & 0.71 & 0.87 \\
\hline \multicolumn{5}{|l|}{ Test-Retest reliability ${ }^{e}$} \\
\hline Mean Difference $^{f}$ & -3.02766 & -.78737 & -1.37505 & -1.44755 \\
\hline ICC & 0.89 & 0.85 & 0.90 & 0.88 \\
\hline
\end{tabular}

difference in SGRQ-CM impact subscale score between dyspnea grade IV vs III patients, as it differentiates the house bound patients from patients who can perform their normal activities. Using anchor-based approach the MCID was calculated as 5.07 (95\% CI -2.54-12.67).

Mean difference (95\% CI) in SGRQ-CM impact and activity subscale scores during hospital admission and after one month of discharge from hospital were displayed in Table 7. Using distribution-based approach MCID was calculated as 6.05 (5.30-6.80).

\section{Discussion}

Our results demonstrated highly acceptable reliability, validity and responsiveness of SGRQ-CM in Malaysian COPD patients. The results are in line with the results of original SGRQ-C results and suggests that the Malaysian version of SGRQ-C is conceptually equal to original

Table 4 Results of correlation between baseline SGRQ-CM scores and other clinical and health status measures

\begin{tabular}{lllll}
\hline & Symptom & Activity & Impact & Total \\
\hline FEV $_{1} /$ FVC & -0.35 & -0.46 & -0.42 & -0.46 \\
FEV $_{1} \%$ predicted & -0.31 & -0.42 & -0.40 & -0.42 \\
CAT & 0.79 & 0.76 & 0.87 & 0.86 \\
EQ-5D-5 L UI & -0.71 & -0.74 & -0.80 & -0.82 \\
EQ-VAS & -0.53 & -0.59 & -0.56 & -0.61 \\
mMRC Dyspnea & 0.64 & 0.79 & 0.63 & 0.72 \\
\hline
\end{tabular}

Results from Pearson correlation Coefficient test; Correlation is significant at the 0.05 level (2-tailed); High \% Predicted FEV1 and EQ-5D-5 L score indicate better quality of life; CAT COPD assessment test; EQ-5D-5 L UI, European Quality of life 5-Dimension 5-Level questionnaire Utility index score; EQ-VAS, European Quality of life 5-Dimension 5-Level questionnaire Visual analogue score; FEV $_{1}$, forced expiratory volume in one second; FVC, Forced vital capacity; $\mathrm{FEV}_{1}(\%)$, percentage predicted $\mathrm{FEV}_{1}$; mMRC modified medical research council dyspnea scale; spirometric data were post-bronchodilator
SGRQ-C and is considered valid to use in Malaysian population. The SGRQ-CM questionnaire and all the three subscales showed good to excellent internal consistency and test retest reliability. As reported in literature, internal consistency is considered good if Cronbach's $\alpha$ is greater than 0.7 [25]. The Cronbach's $\alpha$ coefficient for total and all the subscales was reported above 0.7. The Cronbach's $\alpha$ coefficient values were comparable to published data. It was slightly greater than that reported in similar validation study of Chinese version of SGRQ-C [35]. The Cronbach's $\alpha$ coefficient for Chinese version of SGRQ-C was reported from 0.59 to 0.95 [35]. Cronbach's alpha was slightly lesser than other similar validation study of the Spanish and the Persian versions of SGRQ-C [36, 37]. In the Spanish version of SGRQ-C cronbach's alpha was reported as $(0.72$ to 0.94$)$ and in Persian version it was reported as $(0.699$

Table 5 Mean difference in clinical and health status measures at baseline and after 6 months of follow-up $(n=213)$

\begin{tabular}{llll}
\hline & Mean Difference $(95 \% \mathrm{Cl})$ & Effect Size & $p$ value \\
\hline SGRQ-CM & & & \\
Symptom & $-2.29(-3.14-1.43)$ & 0.38 & $<0.001$ \\
Activity & $-1.51(-2.52-0.48)$ & 0.27 & $<0.01$ \\
Impact & $-2.03(-3.0-1.06)$ & 0.35 & $<0.001$ \\
Total & $-1.73(-2.62-0.84)$ & 0.30 & $<0.001$ \\
Spirometry Values & & \\
FEV $_{1} /$ FVC & $0.54(-0.09-1.18)$ & 0.09 & $0.09^{\mathrm{a}}$ \\
FEV $_{1}(\%)$ & $0.57(-0.17-1.31)$ & 0.10 & $0.13^{\mathrm{a}}$ \\
\hline
\end{tabular}

${ }^{a}$ Mean difference is non-significant; $\mathrm{FEV}_{1}$, forced expiratory volume in one second; FVC, Forced vital capacity; FEV $(\%)$, percentage predicted FEV ${ }_{1}$; Spirometric data were post-bronchodilator; SGRQ-CM, Malaysian version of St George's Respiratory COPD specific Questionnaire 
Table 6 Mean difference in SGRQ-CM scores between patients at different levels of disease severity

\begin{tabular}{|c|c|c|c|c|c|c|c|c|c|}
\hline & \multicolumn{3}{|l|}{ Activity Subscale } & \multicolumn{3}{|l|}{ Impact Subscale } & \multicolumn{3}{|c|}{ SGRQ-CM Total Score } \\
\hline & $\begin{array}{l}\text { Mean Difference } \\
(95 \% \mathrm{Cl})\end{array}$ & Effect Size & $p$ value & $\begin{array}{l}\text { Mean Difference } \\
(95 \% \mathrm{Cl})\end{array}$ & Effect Size & $p$ value & $\begin{array}{l}\text { Mean Difference } \\
(95 \% \mathrm{Cl})\end{array}$ & Effect Size & $p$ value \\
\hline \multicolumn{10}{|c|}{ GOLD Classification } \\
\hline IV vs III & $9.35(0.05-18.64)$ & 0.34 & 0.04 & $12.04(1.45-22.63)$ & 0.45 & 0.02 & $10.78(1.38-20.17)$ & 0.37 & 0.02 \\
\hline |II vs II & $9.51(2.58-16.43)$ & 0.33 & $<0.01$ & $8.91(1.02-16.79)$ & 0.31 & 0.00 & $8.26(1.27-15.26)$ & 0.28 & $<0.01$ \\
\hline \multicolumn{10}{|c|}{ mMRC Dyspnea grade } \\
\hline IV vs III & $14.58(9.33-19.83)$ & 0.58 & $<0.001$ & $5.07(2.54-12.67)$ & 0.37 & 0.19 & 8.59 (2.59-14.59) & 0.43 & $<0.01$ \\
\hline III vs II & 8.89 (3.39-14.39) & 0.39 & $<0.01$ & $10.57(2.60-18.54)$ & 0.49 & 0.01 & $9.86(3.57-16.15)$ & 0.46 & $<0.01$ \\
\hline
\end{tabular}

GOLD Global Initiative for Obstructive Lungs disease; GOLD II, FEV1 50 to 80\% predicted; GOLD III, FEV1 30 to $50 \%$ predicted; GOLD IV, FEV1 < $30 \%$ predicted; mMRC modified medical research council dyspnea scale; SGRQ-CM, Malaysian version of St George's Respiratory COPD specific Questionnaire

to 0.916$)[36,37]$. The small difference in the alpha value shows the possibility of the strong impact of culture on activity and impact subscale.

The SGRQ-CM showed significant correlation with other health status measures in this study. The total SGRQ-CM score showed high correlation, while all subscales showed good correlation with CAT, EQ-5D-5 L and mMRC. Similarly, to the original version of SGRQ$\mathrm{C}$, the activity subscale showed highest correlation with dyspnea scale and impact subscale showed highest correlation with other health status measure [5]. The correlation of SGRQ-C total and subscale scores was stronger with EQ-5D-5 L UI as compared to EQ-VAS. The SGRQ-CM and all subscales showed higher correlation with CAT as compared to the EQ-5D-5 L and mMRC. The highest correlation in impact subscale and CAT shows the specificity of SGRQ-CM to measure the activity limitation specifically due to the COPD [38]. A negative correlation was observed between SGRQ-CM scores and $\mathrm{FEV}_{1} \%$ predicted. Patients with higher predicted FEV $_{1}$ scores reported better QoL as compared to patients with a low predicted $\mathrm{FEV}_{1}$ score. This shows SGRQ-CM can accurately distinguish patients at different disease stages. A significant but low correlation between SGRQ-CM and predicted $\mathrm{FEV}_{1}$ was as per expectations. In COPD patients, literature showed a

Table 7 Mean difference in clinical and health status measures during exacerbation and after one month of treatment $(n=47)$

\begin{tabular}{clll}
\hline & Mean Difference $\mathbf{( 9 5 \% ~ C l )}$ & Effect Size & $\boldsymbol{p}$ value \\
\hline SGRQ-CM & & & \\
Activity & $5.94(5.29-6.58)$ & 0.47 & $<0.001$ \\
Impact & $6.05(5.30-6.80)$ & 0.52 & $<0.001$ \\
Spirometry Values & & \\
FEV1/FVC & $-1.57(-1.85-1.30)$ & 0.18 & $<0.001$ \\
FEV1 (\%) & $-1.58(-1.86-1.31)$ & 0.19 & $<0.001$
\end{tabular}

Exacerbation Patients $\mathrm{n}=47$; difference is significant at 0.05 level; $\mathrm{FEV}_{1}$, forced expiratory volume in one second; FVC, Forced vital capacity; FEV 1 (\%), percentage predicted $\mathrm{FEV}_{1}$; Spirometric data were post-bronchodilator; SGRQCM, Malaysian version of St George's Respiratory COPD specific Questionnaire weak relationship between spirometric values and the original SGRQ-C scores. However, the correlation was comparably higher with SGRQ-CM scores than reported in the previous studies [7, 9]. Activity component showed highest correlation with predicted $\mathrm{FEV}_{1}$. Low correlation between SGRQ-CM and $\mathrm{FEV}_{1} \%$ predicted, than between SGRQ-CM and other health status measures gives a better picture of disease severity under the influence of different factors as compared to clinical symptoms.

A health status measure should be able to discriminate patients, according to severity of disease and evaluate the difference in the QoL over time [39]. The effect size gives the estimate of the power of an instrument to differentiate between patients in different stages of disease severity and to evaluate the impact of an intervention. Despite no significant difference in spirometric values at baseline and 6 months, a significant difference was observed in SGRQ-CM total and subscale scores at baseline and 6 months. These results endorse the previous research, that small deviation in lung function considerably affects the QoL of COPD patients [40]. This shows the sensitivity of SGRQ-CM in detecting the health status change as compared to clinical measures. The ability of a health status measure to detect change in health status in COPD patients is important as, health status is the predictor of mortality and COPD exacerbation in COPD patients [41, 42].

In consistent with the original version of SGRQ-C, the Malaysian version was found to be optimal with three factor structure. In EFA maximum items showed loading in their relevant subscales except few items i.e. the item 7 (If you have a wheeze, is it worse in the morning?) is more related to the symptoms but it showed loading in impact subscale. Similarly item 9.4 showed loading in symptom factor instead of activity factor and item 8 , 11.4, 13.3 and 14 showed loading in symptom subscale instead of impact subscale. But in CFA all the items showed loading in their relevant subscales consistent with the previous research. Moreover, factor loadings 
value $>0.4$ were observed for majority of the items, demonstrating the significant contribution of these items in assessment of health status.

MCID is the minimal difference in outcome measure that clinicians and patients would care about. According to Jaeschke and colleagues a strong MCID should be reflective of patient reported outcomes and clinical findings [43]. Both Anchor-based approach and distributionbased approach were used to calculate MCID which represents patient reported outcomes and clinical findings respectively. Using anchor-based approach and distribution-based approach the MCID was calculated as 5.07 (95\% CI -2.54-12.67) and 6.05 (5.30-6.80) respectively. The MCID reported in our study is comparable to the one reported in literature. In Chinese version of SGRQ-C the MCID was reported as 6.6 (95\%CI - 0.814.1) [7]. MCID calculated by all the parameters was greater than recommended MCID of 4 units calculated for original SGRQ-C [31]. The difference in our reported values and the original SGRQ-C may be due to difference in study population and difference in cultural values of Asian and European population. There is no universally accepted method for calculation of MCID. Utilization of maximum possible perspectives to calculate MCID will enhance the interpretability of the instrument. Moreover, a significant difference in SGRQ-C scores in patients suffering from exacerbation and after one month of discharge from hospital shows that SGRQ-C can reliably identify response to therapy in COPD patients.

Until now, generic QOL questionnaires have been used in clinical trials and other research studies in Malaysian COPD patients. Generic questionnaires are considered as less sensitive to changes in health status, as compared to disease specific questionnaires [17, 44]. This limits their use in clinical trials. To the best of authors' knowledge SGRQ-CM is the first COPD specific QoL instrument to be validated for use in COPD patients in Malaysia. The present study has several strengths. The quality of the study was assessed using COSMIN checklist. Among the ten measurement properties mentioned in COSMIN checklist to assess the good methodological quality of a validation study, nine (internal consistency, reliability, content validity, structural validity, hypotheses testing, cross-cultural validity, criterion validity, responsiveness and interpretability) were reported according to the set standards [45]. The sample size may not be large enough as compared to number of items in the scale to perform psychometric validation, but the sample size used for the current study was higher than most of the previously published research on validation of SGRQ-C [6-9]. Longitudinal design allowed more detailed investigation of SGRQ-C correlation with changing health status over the period of time, including exacerbations. Moreover, utilization of anchor-based approach and distribution-based approach to calculate MCID strengthens the results of current research. Despite several strengths few limitations should be kept in mind before interpreting the results. Although larger sample size was used than previous studies, the sample size was still limited for test-retest reliability. Usually one fifth of the total sample size is recommended for the test-retest reliability. The number of patients included in "test-retest reliability" test was according to the above-mentioned recommendation. The mean age reported in the study is bit higher, but it is in line with the previously published research on COPD in Malaysia [46]. The study was single centered, so the results should be generalized with caution. However, the study population is likely to be representative of general population as Penang Hospital is one of the leading Hospitals of South Malaysia and provides services to the majority of population of South Malaysia.

\section{Conclusion}

In conclusion SGRQ-CM has a strong evidence of validity (construct and concurrent), reliability and responsiveness to disease severity in Malaysian COPD patients. SGRQ-C is a reliable instrument to be used for assessment of overall health status of COPD patients and long term follow-up. It can identify the key areas of health problem that health care professional can explore further during consultation. It can also be used as a reliable QoL measure in future research, including randomized clinical trials, rehabilitation studies, and QoL studies in COPD patients. MCID and responsiveness test results shows that it can reliably assess the impact of an intervention in COPD patients and can be used in clinical practice to measure treatment efficacy for selection of optimal treatment.

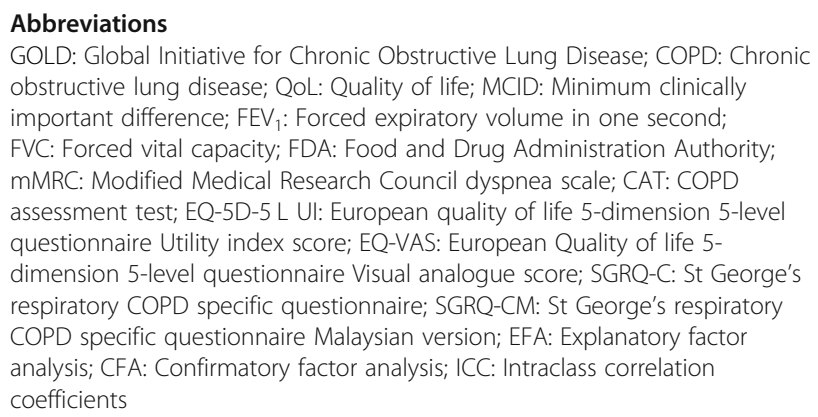

\section{Acknowledgements}

We are thankful to the patients and staff from the Chest Clinic of the Penang Hospital.

\section{Authors' contributions}

AR, MAH, SNH, SA, JM, IHA and RH made substantial contributions to conception and design of the study, and analysis and interpretation of the data. AR and SNH made substantial contributions to analysis and

interpretation of the data. All authors drafted the work or revised it critically 
for important intellectual content. All authors reviewed, critiqued, and approved the final version submitted for publication.

\section{Funding}

No funding was received from any source for the project.

\section{Availability of data and materials}

Data is available on request from corresponding author.

\section{Ethics approval and consent to participate}

The study protocol was approved by the National Medical Research Register Malaysia and clinical research center of concerned hospital (Registration number: NMRR-18-1482-42075). Written informed consent was obtained from all participants.

\section{Consent for publication}

We would like to thank the Director-General of Health, Malaysia for his permission to publish this study.

\section{Competing interests}

The authors declare that they have no conflict of interest.

\section{Author details}

${ }^{1}$ Department of Clinical Pharmacy, School of Pharmaceutical Sciences, University Sains Malaysia, Minden, 11800 Penang, Malaysia. ${ }^{2}$ Faculty of Pharmacy, Bahauddin Zakariya University, Multan, Pakistan. ${ }^{3}$ Department of Pharmacy, Quaid e Azam University, Islamabad, Pakistan. ${ }^{4}$ Respiratory Department, Hospital Pulau Penang, Ministry of Health, Penang, Malaysia.

Received: 7 January 2020 Accepted: 5 May 2020

\section{Published online: 13 May 2020}

\section{References}

1. Global strategy for the diagnosis, management, and prevention of Chronic Obstructive Pulmonary Disease 2019 report. https://goldcopd.org/wpcontent/uploads/2018/11/GOLD-2019-v1.7-FINAL-14Nov2018-WMS.pdf. Accessed Oct 2019.

2. ur Rehman A, Ahmad Hassali MA, Muhammad SA, Shah S, Abbas S, Hyder Ali IAB, Salman A. The economic burden of Chronic Obstructive Pulmonary Disease (COPD) in USA, Europe and Asia: results from a systematic review of the literature. Expert review of pharmacoeconomics \& outcomes research (just-accepted). 2019

3. ur, Rehman A, Hassali MAA, Muhammad SA, Harun SN, Shah S, Abbas S. The economic burden of chronic obstructive pulmonary disease (COPD) in Europe: results from a systematic review of the literature. Eur J Health Econ. 2020;21(2):181-94.

4. Jones P, Quirk F, Baveystock C. Why quality of life measures should be used in the treatment of patients with respiratory illness. Monaldi Arch Chest Dis. 1994;49(1):79-82.

5. Meguro M, Barley EA, Spencer S, Jones PW. Development and validation of an improved, COPD-specific version of the St. George Respir Questionnaire Chest. 2007;132(2):456-63.

6. Lo C, Liang W-M, Hang L-W, Wu T-C, Chang Y-J, Chang C-H. A psychometric assessment of the St. George's respiratory questionnaire in patients with COPD using rasch model analysis. Health Qual Life Outcomes. 2015;13(1): 131.

7. Xu W, Collet J, Shapiro S, Lin Y, Yang T, Wang C, et al. Validation and clinical interpretation of the St George's respiratory questionnaire among COPD patients, China. Int J Tuberc Lung Dis. 2009;13(2):181-9.

8. Morgan BW, Grigsby MR, Siddharthan T, Kalyesubula R, Wise RA, Hurst JR, et al. Validation of the Saint George's respiratory questionnaire in Uganda. BMJ Open Respir Res. 2018;5(1):e000276.

9. Katsoulas TA, Skordilis EK, Myrianthefs P, Theodosopoulou E, Baltopoulos G. Validity of St. George's respiratory questionnaire for Greek patients with chronic obstructive pulmonary disease. Percept Mot Skills. 2010;110(3):772-88.

10. Jones PW. St. George's respiratory questionnaire: MCID. COPD: J Chron Obstruct Pulmon Dis. 2005;2(1):75-9.

11. Mokkink LB, Terwee CB, Patrick DL, Alonso J, Stratford PW, Knol DL, et al. The COSMIN study reached international consensus on taxonomy, terminology, and definitions of measurement properties for health-related patient-reported outcomes. J Clin Epidemiol. 2010;63(7):737-45.
12. Fehnel S, DeMuro C, McLeod L, Coon C, Gnanasakthy A. US FDA patientreported outcome guidance: great expectations and unintended consequences. Expert Rev Pharmacoecon Outcomes Res. 2013;13(4):441-6.

13. Bowden A, Fox-Rushby JA. A systematic and critical review of the process of translation and adaptation of generic health-related quality of life measures in Africa, Asia, Eastern Europe, the Middle East, South America. Soc Sci Med. 2003;57(7):1289-306.

14. Hussain R, Hassali MA, Muneswarao J, Hashmi F. Physicians' understanding and practices of Pharmacovigilance: qualitative experience from A lower middle-income country. Int J Environ Res Public Health. 2020;17(7):2209.

15. Karloh M, Mayer AF, Maurici R, Pizzichini MM, Jones PW, Pizzichini E. The COPD assessment test: what do we know so far?: a systematic review and meta-analysis about clinical outcomes prediction and classification of patients into GOLD stages. Chest. 2016;149(2):413-25.

16. Wacker ME, Jörres RA, Karch A, Wilke S, Heinrich J, Karrasch S, et al. Assessing health-related quality of life in COPD: comparing generic and disease-specific instruments with focus on comorbidities. BMC Pulm Med. 2016;16(1):70.

17. Weldam SW, Schuurmans MJ, Liu R, Lammers J-WJ. Evaluation of quality of life instruments for use in COPD care and research: a systematic review. Int J Nurs Stud. 2013;50(5):688-707.

18. https://www.malaysia.gov.my/portal/statistic (2019). 2019. Accessed Oct 2019.

19. Lai P. Validating instruments of measure: is it really necessary? Malays Fam Physician. 2013;8(1):2.

20. GOLD (2019). Global Initiative for Chronic Obstructive Lung Disease. https:// goldcopd.org/wp-content/uploads/2018/11/GOLD-2019-v1.6-FINAL-08Nov2 018-wms.pdf. 2019.1.1. Accessed May 2018.

21. Jones P, Brusselle G, Dal Negro R, Ferrer M, Kardos P, Levy M, et al. Properties of the COPD assessment test in a cross-sectional European study. Eur Respir J. 2011:38(1):29-35.

22. Mazanec SR, Daly BJ, Douglas SL, Lipson AR. Work productivity and health of informal caregivers of persons with advanced cancer. Res nNurs Health. 2011;34(6):483-95.

23. Shafie AA, Thakumar AV, Lim CJ, Luo N, Rand-Hendriksen K, Yusof FAM. EQ5D-5L valuation for the Malaysian population. Pharmacoeconomics. 2019; 37(5):715-25.

24. Munari AB, Gulart AA, dos Santos K, Venâncio RS, Karloh M, Mayer AF. Modified medical research council dyspnea scale in GOLD classification better reflects physical activities of daily living. Respir Care. 2018;63(1):77-85.

25. Taber KS. The use of Cronbach's alpha when developing and reporting research instruments in science education. Res Sci Educ. 2018;48(6): 1273-96.

26. Koch G. Intraclass correlation coefficient encyclopedia of statistical sciences vol 4 ed S Kotz and NL Johnson. New York: Wiley; 1982.

27. Cohen J. The analysis of variance and covariance: statistical power analysis for the behavioral sciences. Hillsdale: Lawrence Erlbaum Associates; 1988.

28. Li C-H. Confirmatory factor analysis with ordinal data: comparing robust maximum likelihood and diagonally weighted least squares. Behav Res Methods. 2016:48(3):936-49.

29. Tabachnick BG, Fidell LS. Using multivariate statistics, 6th edn. Boston: Pearson; 2013.

30. Jones $P$. Interpreting thresholds for a clinically significant change in health status in asthma and COPD. Eur Respir J. 2002:19(3):398-404.

31. Jones PW, Beeh KM, Chapman KR, Decramer M, Mahler DA, Wedzicha JA. Minimal clinically important differences in pharmacological trials. Am J Respir Crit Care Med. 2014;189(3):250-5.

32. Leidy NK, Wilcox TK, Jones PW, Roberts L, Powers JH, Sethi S. Standardizing measurement of chronic obstructive pulmonary disease exacerbations: reliability and validity of a patient-reported diary. Am J Respir Crit Care Med. 2011;183(3):323-9.

33. Ozalevli S, Ucan ES. The comparison of different dyspnoea scales in patients with COPD. J Eval Clin Pract. 2006:12(5):532-8.

34. Tinsley HE, Brown SD. Handbook of applied multivariate statistics and mathematical modeling: Academic Press; 2000.

35. Chan SL, Chan-Yeung MM, Ooi GC, Lam CL, Cheung TF, Lam WK, et al. Validation of the Hong Kong Chinese version of the St. George respiratory questionnaire in patients with bronchiectasis. Chest. 2002;122(6):2030-7.

36. Ferrer $\mathrm{M}$, Alonso J, Prieto L, Plaza V, Monsó E, Marrades $\mathrm{R}$, et al. Validity and reliability of the St George's respiratory questionnaire after adaptation to a different language and culture: the Spanish example. Eur Respir J. 1996:9(6): 1160-6. 
37. Tafti SF, Cheraghvandi A, Mokri B, Talischi F. Validity and specificity of the Persian version of the Saint George respiratory questionnaire. J Asthma. 2011;48(6):589-92.

38. Wilson IB, Cleary PD. Linking clinical variables with health-related quality of life: a conceptual model of patient outcomes. Jama. 1995;273(1):59-65.

39. Hajiro T, Nishimura K, Tsukino M, Ikeda A, Koyama H, Izumi T. Comparison of discriminative properties among disease-specific questionnaires for measuring health-related quality of life in patients with chronic obstructive pulmonary disease. Am J Respir Crit Care Med. 1998;157(3):785-90.

40. Wise RA. The value of forced expiratory volume in 1 second decline in the assessment of chronic obstructive pulmonary disease progression. Am J Med. 2006;119(10):4-11.

41. Halpin DM, Peterson S, Larsson TP, Calverley PM. Identifying COPD patients at increased risk of mortality: predictive value of clinical study baseline data. Respir Med. 2008:102(11):1615-24.

42. ur, Rehman A, Hassali MAA, Abbas S, Ali IABH, Harun SN, Muneswarao J, et al. Pharmacological and non-pharmacological management of COPD; limitations and future prospects: a review of current literature. J Public Health. 2019:1-10.

43. Jaeschke R, Singer J, Guyatt GH. Measurement of health status: ascertaining the minimal clinically important difference. Control Clin Trials. 1989;10(4): 407-15.

44. ur Rehman A, Naeem F, Abbas S, Ashfaq F, Hassali MAA. Utilization of short message service (SMS) in non-pharmacological management of hypertension. A pilot study in an URBAN public hospital of Multan. Pakistan J Public Health. 2019;27(5):561-7.

45. Mokkink LB, Terwee CB, Patrick DL, Alonso J, Stratford PW, Knol DL, et al. The COSMIN checklist for assessing the methodological quality of studies on measurement properties of health status measurement instruments: an international Delphi study. Qual Life Res. 2010;19(4):539-49.

46. Rehman U, Hassali M, Muhammad S, Shakeel S, Chin O, Ali I, et al. Economic burden of chronic obstructive pulmonary disease patients in Malaysia: A longitudinal study. Pharmacoecon Open. 2020.

\section{Publisher's Note}

Springer Nature remains neutral with regard to jurisdictional claims in published maps and institutional affiliations.

Ready to submit your research? Choose BMC and benefit from:

- fast, convenient online submission

- thorough peer review by experienced researchers in your field

- rapid publication on acceptance

- support for research data, including large and complex data types

- gold Open Access which fosters wider collaboration and increased citations

- maximum visibility for your research: over $100 \mathrm{M}$ website views per year

At $\mathrm{BMC}$, research is always in progress.

Learn more biomedcentral.com/submissions 